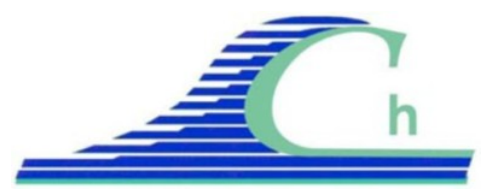

XII ${ }^{\text {ìmes }}$ Journées Nationales Génie Côtier - Génie Civil

Cherbourg, 12-14 juin 2012

DOI:10.5150/jngcgc.2012.096-S @ Editions Paralia CFL

disponible en ligne - http://www.paralia.fr - available online

\title{
Plan d'action pour la protection des cordons littoraux et marais maritimes associés en rade de Brest
}

\author{
Pierre STEPHAN $^{1}$, Serge SUANEZ ${ }^{2}$, Bernard FICHAUT ${ }^{2}$
}

1. Laboratoire de Géographie Physique - UMR 8591 CNRS,

Universités Paris 1 Panthéon Sorbonne et Paris 12,

1 Place Aristide Briand, 92195 Meudon cedex, France.

pierre.stephan@cnrs-bellevue.fr

2. LETG-Géomer-Brest, UMR 6554 CNRS,

Institut Universitaire Européen de la Mer, Université de Brest,

Technopôle Brest-Iroise, Place Nicolas Copernic, 29280 Plouzané, France.

Serge.suanez@univ-brest.fr ; Bernard.fichaut@univ-brest.fr

\section{Résumé :}

La présente étude vise à définir les sites d'intervention et les actions prioritaires pour la mise en œuvre de mesures de conservation et de restauration des cordons littoraux et des marais maritimes associés situés en rade de Brest. Une cinquantaine de cordons, répartis sur 43 sites, ont fait l'objet d'une évaluation patrimoniale à partir de critères géomorphologiques et écologiques (flore, habitat, faune). Les principales causes de dégradation de ce patrimoine qui nécessiteraient une intervention sont : (i) l'érosion des cordons, (ii) la progression de la spartine alterniflore et la perte de la biodiversité des marais maritimes, (iii) la fréquentation anthropique. Le croisement de la valeur patrimoniale et des types de dégradation permet d'identifier une dizaine de secteurs exigeant des interventions prioritaires.

\section{Mots-clés :}

Cordon littoral - Marais maritime - Rade de Brest - Gestion littorale

\section{Introduction}

La rade de Brest compte un nombre étonnant de cordons littoraux d'une grande diversité morphologique. Déjà souligné par GUILCHER et al. (1957), cet ensemble constitue un remarquable patrimoine géomorphologique auquel sont bien souvent associés des marais maritimes à très forte valeur écologique. D'un point de vue floristique, ces marais comptent plusieurs espèces rares ou menacées, comme le petit statice (Limonium humile), une lavande de mer endémique que l'on ne retrouve en France que dans quelques anses de la rade (QUÉRÉ, 2009). Depuis quelques décennies, cette espèce végétale est peu à peu supplantée par une herbacée invasive d'origine américaine, la spartine alterniflore (Spartina alterniflora), dont la progression est très rapide sur les schorres et les slikkes de la rade (SPARFEL et al., 2005). Ces dernières années, la prise de conscience grandissante de la nécessité de protéger ces milieux face 
à la perte de biodiversité, la mise en place des Zones Natura 2000 (Elorn et rade) et la poursuite d'un contrat multi-partenarial en rade de Brest (Contrat de rade) a permis de mobiliser les collectivités territoriales et plusieurs acteurs locaux sur la question de la conservation des cordons littoraux et des marais associés. Cette étude constitue l'étape préalable à la mise en place d'un Contrat Nature, outil de gestion permettant d'engager, dès 2012, une série d'action de protection et restauration des milieux naturels littoraux dans ce secteur. Ce travail a eu pour objectifs de : (i) dresser l'inventaire et la typologie des cordons et de leur marais maritimes, (ii) retracer la dynamique des milieux et le rôle des forçages naturels et anthropiques dans ces évolutions, (iii) définir les enjeux patrimoniaux et estimer la valeur géomorphologique et écologique des sites d'étude, (iv) cerner les causes de dégradation de ce patrimoine naturel et définir les priorités d'action ainsi que les sites sur lesquels il convient d'intervenir rapidement.

\section{Matériel et méthode}

\subsection{Mobilité récente des cordons littoraux}

La mobilité récente de 24 cordons littoraux, ainsi que la dynamique végétale de cinq marais maritimes envahis par la spartine alterniflore, ont été retracées à partir d'une analyse diachronique de 200 photographies aériennes de l'IGN et de levés réalisés au DGPS sur la période 1948-2011 (SPARFEL et al., 2005; STÉPHAN, 2011). La méthode de travail repose sur la numérisation des clichés, leur correction géométrique, leur géoréférencement, leur intégration dans un SIG avec une marge d'erreur estimée à $\pm 1 \mathrm{~m}$. Pour les cordons littoraux, la limite de végétation supralittorale a été numérisée et les évolutions ont été quantifiées le long de transects perpendiculaires au rivage. Pour les marais maritimes, les tâches de spartine ont été numérisées et les superficies ont été calculées.

\subsection{Rôle des forçages météo-marins et anthropiques}

Le rôle des événements météo-marins dans la mobilité des cordons littoraux a été évalué en dressant la chronologie des épisodes de franchissement des cordons (STÉPHAN, 2008). Les niveaux d'eau extrêmes à la côte ont été déterminés à partir (i) des niveaux d'eau enregistrés par le marégraphe de Brest, (ii) et de l'estimation conjointe du wave-setup et du swash-runup d'après les formules de STOCKDON et al. (2006). Les données de vagues à la côte (Hs et Tp) ont été calculées sur chacun des sites d'étude à partir des valeurs de vent enregistrées à la station Météo-France de LanvéocPoulmic en utilisant les équations de DONELAN et al. (1992), testées et validées par des mesures de houles in-situ effectuées dans le port-abri de Brest. Les forçages anthropiques ont été étudiés de façon essentiellement descriptive, à travers une observation des photographies aériennes, permettant de retracer l'historique des équipements côtiers à proximité des secteurs d’étude. 


\section{XII ${ }^{\text {èmes }}$ Journées Nationales Génie Côtier - Génie Civil \\ Cherbourg, 12-14 juin 2012}

\subsection{Méthode d'évaluation patrimoniale}

L'évaluation patrimoniale s'est attachée à déterminer conjointement la valeur géomorphologique et écologique (flore, habitats, faune) des cordons et des marais maritimes associés, selon un indice compris entre 0 et 100. Les critères, les souscritères, les indicateurs et leur poids respectif, ainsi que l'échelle et les principes de notation ont été définis en concertation avec collectivités locales, les botanistes du Conservatoire Botanique National de Brest, des ornithologues du Parc Régional d'Armorique, des géomorphologues (tableau 1).

Tableau 1. Critères d'évaluation patrimoniale appliqués aux cordons littoraux et aux marais associés de la rade de Brest. Le poids de chaque critère dans la définition de l'indice est donné entre parenthèses.

\begin{tabular}{|c|c|c|c|c|c|}
\hline Critères & Sous-critères & Indicateurs & $\begin{array}{l}\text { Données } \\
\text { utilisées }\end{array}$ & $\begin{array}{l}\text { Echelle de } \\
\text { notation }\end{array}$ & $\begin{array}{l}\text { Critères de } \\
\text { notation }\end{array}$ \\
\hline \multirow{3}{*}{$\begin{array}{l}\text { Géomorphologiques } \\
(50 / 100)\end{array}$} & $\begin{array}{l}\text { Intérêt géomorphologique } \\
\text { principal }(25 / 100)\end{array}$ & $\begin{array}{l}\text { Dimension, dynamique, état de } \\
\text { dégradation des cordons littoraux } \\
\text { et des marais maritimes }\end{array}$ & $\begin{array}{l}\text { STEPHAN (2011) } \\
\text { SPARFEL et al. (2005) }\end{array}$ & de $0 \dot{a} 3$ & $\begin{array}{l}\text { 0: anthropisé } \\
\text { 1: faible } \\
\text { 2: important } \\
\text { 3: majeur }\end{array}$ \\
\hline & $\begin{array}{l}\text { Intérêt géomorphologique } \\
\text { secondaire }(5 / 100)\end{array}$ & $\begin{array}{l}\text { Présence de stratigraphies, de dépôts } \\
\text { sédimentaires, de formes fossiles }\end{array}$ & STEPHAN (2011) & de $0 \grave{a} l$ & $\begin{array}{l}\text { 0: Absence } \\
\text { I: Présence }\end{array}$ \\
\hline & $\begin{array}{l}\text { Rareté à l'échelle de la } \\
\text { Bretagne }(20 / 100)\end{array}$ & $\begin{array}{l}\text { Type de morphologie, occurence sur le } \\
\text { littoral régional }\end{array}$ & STEPHAN (2011) & de $0 \grave{a} 3$ & $\begin{array}{l}\text { 0: anthropisé } \\
\text { 1: commun } \\
\text { 2: rare } \\
\text { 3: très rare }\end{array}$ \\
\hline \multirow{11}{*}{$\begin{array}{l}\text { Ecologiques } \\
(50 / 100)\end{array}$} & \multirow{2}{*}{$\begin{array}{l}\text { Flore } \\
(16,7 / 100)\end{array}$} & $\begin{array}{l}\text { Espèce végétale protégée } \\
(12 / 100)\end{array}$ & \multirow{2}{*}{ QUERE (2008) } & de $0 \dot{a} l$ & $\begin{array}{l}\text { 0: Absence } \\
\text { I: Présence }\end{array}$ \\
\hline & & $\begin{array}{l}\text { Espèce floristique rare à l'échelle } \\
\text { régionale (5/I00) }\end{array}$ & & de $0 \dot{a} l$ & $\begin{array}{l}\text { 0: Absence } \\
\text { 1: Présence }\end{array}$ \\
\hline & \multirow{3}{*}{$\begin{array}{l}\text { Habitats } \\
(16,7 / 100)\end{array}$} & $\begin{array}{l}\text { Diversité : nombre d'habitats d'intérêt } \\
\text { communautaire } \\
(7 / 100)\end{array}$ & \multirow{3}{*}{$\begin{array}{l}\text { Base de données carto- } \\
\text { graphiques Natura } 2000 \\
\text { BOUGAULT et al. (2004) }\end{array}$} & de $0 \dot{a} 3$ & $\begin{array}{l}\text { 0: Absence } \\
\text { 1: Nbre }[1 ; 2] \\
\text { 2: Nbre }[3 ; 4] \\
\text { 3: Nbre }>4\end{array}$ \\
\hline & & $\begin{array}{l}\text { Richesse : part des habitats d'intérêt } \\
\text { communautaire dans la superficie totale } \\
\text { des marais maritimes et cordons littoraux } \\
(7 / 100)\end{array}$ & & de $0 \grave{a} 3$ & $\begin{array}{l}\text { 0: Absence } \\
\text { 1: }[1-30 \% \mid \\
\text { 2: }[30-60 \%[ \\
\text { 3: }>60 \%\end{array}$ \\
\hline & & $\begin{array}{l}\text { Habitats d'intérêt régional (méga- } \\
\text { phorbiaie, roselière,...) au titre de la } \\
\text { protection des zones humides }(2,7 / 100)\end{array}$ & & de $0 \dot{a} l$ & $\begin{array}{l}\text { 0: Absence } \\
\text { l: Présence }\end{array}$ \\
\hline & \multirow{6}{*}{$\begin{array}{l}\text { Avifaune } \\
(16,7 / 100)\end{array}$} & $\begin{array}{l}\text { Cordon littoral : zone de nidification } \\
(2,7 / 100)\end{array}$ & \multirow{6}{*}{ MOREL et al. (2005) } & de 0 à 1 & $\begin{array}{l}\text { 0: Absence } \\
\text { 1: Présence }\end{array}$ \\
\hline & & $\begin{array}{l}\text { Cordon littoral : zone de reposoir pour } \\
\text { les anatidés }(2,7 / / 00)\end{array}$ & & de $0 \dot{a} l$ & $\begin{array}{l}\text { 0: Absence } \\
\text { I: Présence }\end{array}$ \\
\hline & & $\begin{array}{l}\text { Cordon littoral : zone de reposoir pour } \\
\text { les limicoles }(2,7 / 100)\end{array}$ & & de $0 \dot{a} I$ & $\begin{array}{l}\text { 0: Absence } \\
\text { I: Présence }\end{array}$ \\
\hline & & $\begin{array}{l}\text { Marais maritime : zone d'alimentation et } \\
\text { de reposoir pour les anatidés }(2,7 / 100)\end{array}$ & & de $0 \grave{a} l$ & $\begin{array}{l}\text { 0: Absence } \\
\text { l: Présence }\end{array}$ \\
\hline & & $\begin{array}{l}\text { Marais maritime: zone d'alimentation et } \\
\text { de reposoir pour les limicoles }(2,7 / 100)\end{array}$ & & de $0 \dot{a} I$ & $\begin{array}{l}\text { 0: Absence } \\
\text { 1: Présence }\end{array}$ \\
\hline & & $\begin{array}{l}\text { Présence de passereaux paludicoles } \\
(2,7 / 100)\end{array}$ & & de $0 \dot{a} 1$ & $\begin{array}{l}\text { 0: Absence } \\
\text { 1: Présence }\end{array}$ \\
\hline
\end{tabular}

La valeur géomorphologique a été établie en fonction de (i) la dimension, la dynamique et l'état de dégradation des formes (intérêt géomorphologique principal), (ii) la présence de dépôts quaternaires susceptibles de renseigner sur les paléo-environnements littoraux (intérêt géomorphologique secondaire), (iii) la rareté de ces formes à l'échelle régionale, en s'appuyant sur les études de SPARFEL et al. (2005) et STEPHAN (2011). La valeur écologique se fonde sur (i) la présence d'une flore rare et/ou protégée (QUÉRÉ et al., 
2008), (ii) la diversité, la richesse et la présence d'habitats d'intérêt communautaire et/ou régional, déterminées d'après la base de données cartographiques Natura 2000 des secteurs de l'Elorn, de la rade de Brest et de la presqu'île de Crozon (BOUGAULT et al., 2004), (iii) la présence d'une avifaune d'intérêt communautaire (MOREL et al., 2005).

\section{Résultats}

\subsection{Inventaire et typologie des milieux}

L’inventaire des cordons littoraux permet de recenser une cinquantaine d'accumulations, réparties sur 43 sites (figure 1).

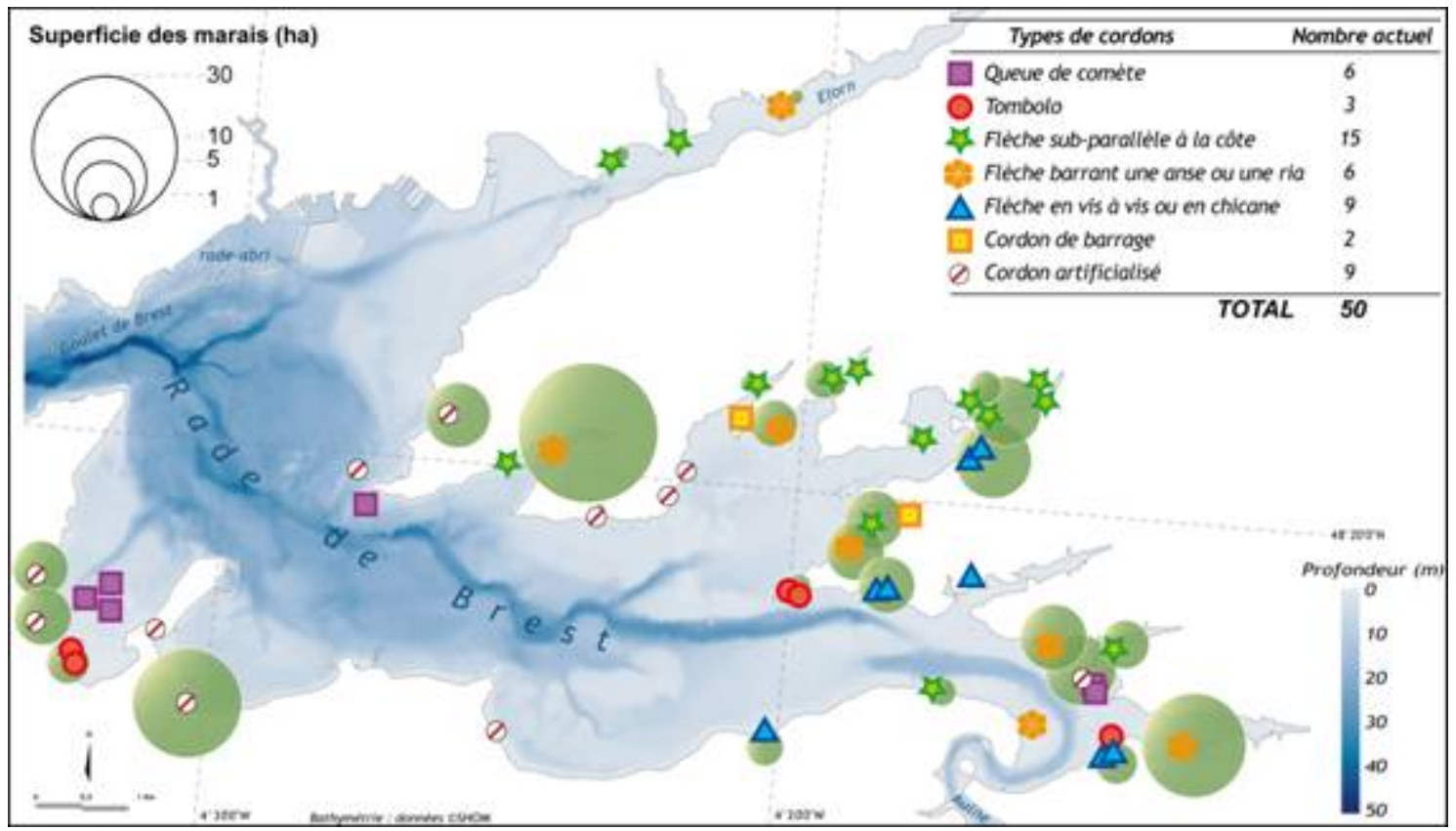

Figure 1. Typologie des cordons littoraux et superficie des marais maritimes associés.

Cette grande richesse se double également d'une très forte diversité morphologique. En reprenant la typologie morphologique dressée par GUILCHER et al. (1957), la rade compte des queues de comète, des tombolos simples et doubles, des flèches subparallèles à la côte, des flèches de mi-baie, des flèches en vis-à-vis et en chicane, des cordons de barrage ainsi que des flèches totalement artificialisées. Les marais de la rade abritent quatre espèces floristiques protégées à l'échelle nationale et sept espèces végétales rares au niveau régional (QUÉRÉ et al., 2008). D’un point de vue faunistique, cet espace est également un site d'hivernage d'importance internationale pour l'avifaune. La population d'oiseaux d'eau s'élève à 20000 individus en hiver (MOREL et al., 2005). On y recense 34 espèces d'intérêt européen dont une grande partie 


\section{XII ${ }^{\text {èmes }}$ Journées Nationales Génie Côtier - Génie Civil \\ Cherbourg, 12-14 juin 2012}

(anatidés, limicoles et passereaux paludicoles) fréquentent les cordons et les marais en tant que zone de repos ou de nourrissage. Enfin, cette biodiversité animale et végétale bénéficie d'habitats riches et diversifiés. Sept habitats d'intérêt communautaire différents peuvent être identifiés (BOUGAULT et al., 2004). Ils représentent en moyenne $53 \%$ de la superficie totale de nos sites d'étude et couvrent 122 ha.

\subsection{Dynamique des cordons littoraux et des marais maritimes}

Sur les 24 cordons littoraux étudiés, les trois quarts ont manifesté une tendance au recul au cours des six dernières décennies (STÉPHAN, 2011). L'analyse des épisodes de franchissement montre que les forçages météo-marins sont responsables de ce recul principalement sur les sites exposés à de grandes longueurs de fetch. Ailleurs, les phénomènes d'érosion résultent d'une sous-alimentation sédimentaire, car les falaises meubles qui constituent la principale source d'apport ne semblent plus livrer autant de matériel que par le passé (STÉPHAN, 2011). Les pressions anthropiques le long du rivage (blocage des transits par les cales et les épis, prélèvements sédimentaires, artificialisation des milieux, surfréquentation) ont également contribué à aggraver cette situation. Dans les marais maritimes, les principales évolutions sont liées à la progression très rapide de la spartine alterniflore au cours des quatre dernières décennies, à des vitesses estimées à 400 m²/an à Pont-Callec, 1300 m²/an au Pédel, 825 $\mathrm{m}^{2} / \mathrm{an}$ dans le marais de Mengleuz, $1400 \mathrm{~m}^{2} / \mathrm{an}$ dans celui de Kérroullé et $1700 \mathrm{~m} / \mathrm{an}$ dans le marais du Faou. Les travaux de SPARFEL et al. (2005) n'ont pas permis d'éclaircir les raisons d'une telle progression.

\subsection{Classement patrimonial}

La figure 2 présente le classement des différents sites d'étude en fonction de leur valeur patrimoniale. Ce classement permet de distinguer les sites d'intérêt patrimonial :

- très fort, dont l'indice est compris entre 80 et 100 (100 étant le maximum théorique) ;

- fort, dont l'indice est compris entre 50 et 80 ;

- modéré, dont l'indice est compris entre 30 et 50 ;

- faible, dont l'indice est inférieur à 30.

\subsection{Les causes de dégradation des sites}

Trois causes de dégradation des sites d'études ont été identifiées. Tout d'abord, l'érosion des cordons littoraux par les vagues conduit à leur démantèlement progressif et, à terme, à la dégradation des marais maritimes situés en arrière. Ensuite, la progression de la spartine alterniflore sur les schorres des marais entraîne une perte de biodiversité. En effet, l'homogénéisation des milieux se solde par une diminution de la diversité et de la richesse des habitats, par la disparition la flore vulnérable comme le petit statice, et conduit à une diminution des aires de nourrissage des limicoles et autres oiseaux d'estrans. Enfin, la surfréquentation de certains sites se traduit par l'érosion des 
cordons, la détérioration des habitats et le dérangement de l'avifaune. Cette pression est très forte en rade où de nombreuses cales permettent aux véhicules motorisés d'accéder facilement à l'estran. La création récente de nouveaux sentiers côtiers de randonnées dans des secteurs jusqu’à présent peu fréquentés pose également question.

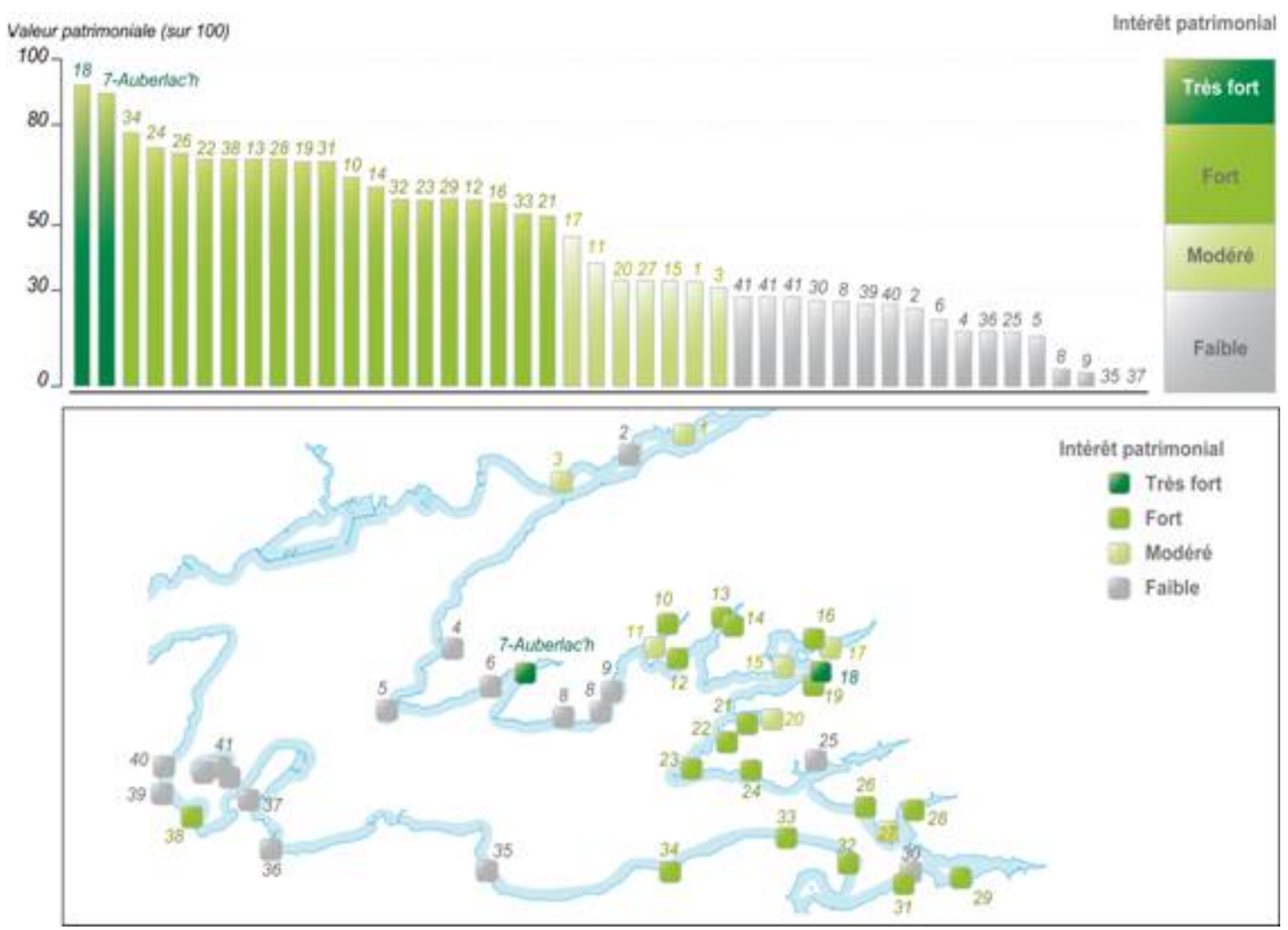

Figure 2. Classement des sites en fonction de leur valeur patrimoniale.

\subsection{Les priorités d'action}

Trois actions prioritaires sont donc à préconiser sur les sites présentant les plus fortes valeurs patrimoniales. La première action vise à limiter l'érosion des cordons soumis à un recul rapide par l'emploi de méthodes "douces" basées sur du rechargement, du colmatage de brèche ou des opérations ponctuelles de reprofilage. Ce type d'opération a d'ores et déjà été mené ponctuellement en rade et a donné des résultats satisfaisants (STÉPHAN, 2011). Les ouvrages perméables aux transits sédimentaires de type "by pass" sont également conseillés afin d'éliminer le blocage du matériel contre les cales d'accès à l'estran et les jetées portuaires. La seconde action vise à réduire la progression de la spartine alterniflore dans les marais les plus touchés par le phénomène. Toutefois, les travaux de SPARFEL et al. (2005) ont montré que quel que soit le type d'opération envisagé (traitement par herbicides, fauchage, introduction d'insectes phytophages), l'éradication complète de la spartine reste difficile lorsque la plante est installée sur de grandes superficies. Les expériences effectuées ces dernières années par le 


\section{XII ${ }^{\text {èmes }}$ Journées Nationales Génie Côtier - Génie Civil \\ Cherbourg, 12-14 juin 2012}

Conservatoire botanique indiquent que seuls l'arrachage et de bâchage des tâches pionnières donnent des résultats satisfaisants dans les secteurs encore préservés (QUÉRÉ, 2009). La troisième action prioritaire cherchera à réduire les impacts d'une fréquentation excessive des sites par les randonneurs et les automobilistes, en restreignant les accès à l'estran et en prévoyant de nouveaux itinéraires de randonnée.

\section{Discussion}

A ce jour, il est rare que les méthodes d'évaluation patrimoniale mises en œuvre sur les sites naturels côtiers tiennent compte conjointement des aspects géomorphologiques et écologiques. Généralement, seuls les caractéristiques écologiques sont considérées, car elles s'appuient sur des données floristiques ou faunistiques plus communément quantifiées et/ou quantifiables. En ce sens, le classement patrimonial proposé dans le cadre de cette étude est original, même s'il reste difficilement transposable à d'autres secteurs côtiers. En effet, les critères et les indicateurs retenus, ainsi que leur poids respectif dans l'évaluation patrimoniale sont le résultat de choix relativement arbitraires, effectués dans le cadre d'une concertation locale avec les seuls acteurs du littoral de la rade de Brest. En outre, ces choix aboutissent à une évaluation relativement simpliste de milieux naturels dont la nature et le fonctionnement sont beaucoup plus complexes et pour lesquels les données biogéophysiques sont souvent incomplètes. Les mesures conservatoires mises en œuvre s'appuient alors sur une connaissance partielle des milieux naturels et peuvent s'avérer inadaptées ou inappropriées. Dans cette étude, les principales lacunes concernent la faune fréquentant les cordons et les marais de la rade, qui n'est connue qu'à travers les populations d'oiseaux.

L'une des pistes d'amélioration de ce travail consisterait en l'utilisation d'une méthode d'Analyse Multicritère (AMC) d'aide à la décision de type ELECTRE (MAYSTRE et al., 1994), utilisée dans les études environnementales lorsqu'il s'agit d'agréger de nombreux indicateurs de nature très différente pour aboutir à un classement permettant de prioriser les choix de gestion ou de conservation. Cette méthode permettrait de rendre notre démarche d'évaluation patrimoniale moins subjective et plus facilement transposable à d'autres secteurs naturels côtiers.

\section{Conclusions}

Dès la fin 2012, une série d'interventions devrait être mise en œuvre sur certains cordons littoraux et les marais maritimes de la rade, dans le cadre d'un programme pluriannuel d'actions de préservation et/ou de restauration de sites naturels de Bretagne, appelé "Contrat Nature". Si les sites d'intervention et les actions prioritaires sont désormais bien définis, un important travail de concertation reste à mener entre le maître d'ouvrage des opérations (Région Bretagne et Brest Métropole Océane) et les différents acteurs et propriétaires fonciers. De plus, le nombre d'actions et leur coût seront limités, les Contrats Nature bénéficiant d'un financement réduit ( $40 \mathrm{~K} € / \mathrm{an}$ ) pour une période 
limitée à 3 ans. Enfin, certaines difficultés techniques liées à l'accessibilité des sites, à leurs statuts de protection ou au niveau de dégradation plus ou moins avancé du milieu devront être surmontées pour mener à bien les différentes opérations.

\section{Références bibliographiques}

BOUGAULT C., HARDEGEN M., QUÉRÉ E. (2004). Site Natura 2000 n46. Rade de Brest, Estuaire de l'Aulne. Inventaire et cartographie des habitats terrestres et des espèces végétales d'intérêt communautaire. Conservatoire Botanique National de Brest, Direction Régionale de l'Environnement, 177 p.

DONELAN M.A., SKAFEL M., GRABER H., LIU P., SCHWAB D., VENKATESH S. (1992). On the growth rate of wind-generated waves. Atmosphere-Ocean, vol. 30, $\mathrm{n}^{\circ} 3$, pp 457-478. doi:10.1080/07055900.1992.9649449

GUILCHER A., VALLANTIN P., ANGRAND J.-P., GALLOY P. (1957). Les cordons littoraux de la rade de Brest. Cahiers Océanographiques, $\mathrm{n}^{\circ}$ 1, pp 21-54.

MAYSTRE L.-Y., PICTET J., SIMOS J. (1994). Méthodes multicritères ELECTRE, description, conseils pratiques, cas d'application à la gestion environnementale, Presses polytechniques et universitaires romandes, collection Gérer l'environnement, $323 \mathrm{p}$.

MOREL R., CADIOU B., DEPONTALLIER L., BARGAIN B. (2005). Evaluation des zones de Protection Spéciale du Finistère et des Côtes d'Armor. Rapport Bretagne Vivante - SEPNB, DIREN Bretagne, $247 \mathrm{p}$.

QUÉRÉ E., MAGNAON S., RAGOT R., GAGER L., HARDY F. (2008). Atlas de la flore du Finistère. Flore vasculaire. Siloë, Nantes, 693 p.

QUÉRÉ E. (2009). Vingt ans de suivi et de conservation du Limonium humile Miller en rade de Brest. Bilan 2009. Conservatoire Botanique National de Brest, Brest Métropole Océane, 96 p.

SPARFEL L., FICHAUT B., SUANEZ S. (2005). Progression de la Spartine (Spartina alterniflora Loisel) en rade de Brest (Finistère) entre 1952 et 2004 : de la mesure à la réponse gestionnaire. Norois, $\mathrm{n}^{\circ}$ 196, pp 109-123.

STÉPHAN P. (2008). Mobilité des cordons littoraux et submersions marines. Les flèches de galets de la rade de Brest (Bretagne, France). European Journal of Environmental and Civil Engineering. vol. 12 (1-2), pp 161-178. doi:10.3166/ejece.12.161-178 STÉPHAN P. (2011). Quelques données nouvelles sur la mobilité récente et le bilan sédimentaire des flèches de galets de Bretagne. Géomorphologie : Relief. Processus. Environnement, $\mathrm{n}^{\circ} 2$, pp 205-232.

STOCKDON H.F., HOLMAN R.A., HOWD P.A., SALLENGER A.H. (2006). Empirical parameterization of setup, swash and runup. Coastal Engineering, $\mathrm{n}^{\circ} 53$, pp 573-588. doi:10.1016/j.coastaleng.2005.12.005 